\title{
A Virtualization Approach to Correct Systematic Errors in Measured TDOA and FDOA for Low Orbit Dual-Satellite Positioning Systems
}

\author{
Gang Li $\mathbb{D}^{1,2}$ Min Zhou, ${ }^{3}$ Hongwen Tang, ${ }^{4}$ and Hongbin Chen ${ }^{5}{ }^{5}$ \\ ${ }^{1}$ Wuhan Textile University, School of Electronic and Electrical Engineering, Wuhan, Hubei 430200, China \\ ${ }^{2}$ Concordia University, Department of Electrical and Computer Engineering, Montreal, QC H4B 1R6, Canada \\ ${ }^{3}$ South Sagittarius Integration Co. LTD, Wuhan, Hubei 430056, China \\ ${ }^{4}$ South-central University for Nationalities, College of Electronics and Information Engineering, Wuhan, Hubei 430072, China \\ ${ }^{5}$ Guilin University of Electronic Technology, Key Laboratory of Cognitive Radio and Information Processing, \\ Guilin 541004, China
}

Correspondence should be addressed to Hongbin Chen; chbscut@guet.edu.cn

Received 3 May 2021; Revised 7 July 2021; Accepted 16 August 2021; Published 6 September 2021

Academic Editor: Giuseppe Torrisi

Copyright $\odot 2021$ Gang Li et al. This is an open access article distributed under the Creative Commons Attribution License, which permits unrestricted use, distribution, and reproduction in any medium, provided the original work is properly cited.

The low-orbit dual-satellite passive location system provides a cost-efficient and easy implementation platform, by which positions of unknown emitters on the Earth can be determined through measuring both the time and the frequency differences by two loworbit satellites in space. However, in reality, this dual-satellite location system has low positioning accuracy because of the existence of systematic errors. In this paper, in order to address the problem of low positioning accuracy in low-orbit dual-satellite systems, a virtualization approach, consisting of the establishment of the virtual reference station and virtual frequency conversion, is proposed to correct systematic errors in the system. Specifically, we first analyze the coming source of systematic errors in the dual-satellite location system, and then, a virtual reference station and virtual frequency are constructed to correct errors in the measured time difference of arrival and the frequency difference of arrival, respectively. Simulation results show that systematic errors caused by the measured time difference of arrival can be significantly reduced, and the correction efficiency, defined as a ratio between remaining errors after implementing the proposed method over uncorrected ones, for the measured frequency difference of arrival, largely relies on both the virtual frequency and the transmission frequency of reference stations.

\section{Introduction}

Satellites for precise positioning of unknown radiative targets on the ground, such as radars, sonars, and tracking radiative objectives, are of great significance in the space information confrontation $[1,2]$. Unlike active location systems, such as the radar and sonar, the passive location technologies were commonly adopted to detect and locate unknown targets because this location model only subjectively receives signals from unknown targets to obtain their location information, which enhances the concealment and further protects itself. In passive location systems, the position of unknown targets can be obtained by measuring the Doppler frequency for multiple times by using a single satellite platform [3]. Unfortunately, the positioning accuracy by this method is very low, and it can be suitable for stationary targets. Alternatively, a foursatellite passive location system [4] was proposed by only measuring the time difference of arrival (TDOA). Even though this method can provide a more accurate positioning result, the costs to build up and maintain this platform is a bit higher as four satellites are needed. As a consequence, the low-orbit dual-satellite location system by measuring both the TDOA and the frequency difference of arrival (FDOA) has obtained numerous attention from both industries and academia, as this positioning system only needs double adjacent satellites, and the positioning accuracy is higher than that by the single satellite platform 
$[5,6]$. However, because of the existence of systematic errors in the measured TDOA and FDOA value, which probably resulted from the transponder delay, the frequency shift, and other unknown factors, the positioning accuracy of this system is generally in the range of $3-10 \mathrm{~km}$ [7-9]. In comparison to the four-satellite location system, the positioning accuracy of the dual-satellite location system is relatively poor and needed to be improved. In literature, although numerous previous efforts $[7,10,11]$ have developed the efficient positioning algorithms and provided positioning accuracy analysis for the dual-satellite location system, limited research focused on eliminating systematic errors so as to improve the positioning accuracy. For example, a method based on ephemeris correction technique to amend the errors of measured TDOA and FDOA was put forward in $[12,13]$. However, this method needed at least four reference stations, and the correction performance largely relied on the layout of the reference stations. Moreover, the correction for ephemeris errors was only considered in this paper. As a result, this method will limit its application in practice. Therefore, to develop a method for correcting systematic errors in both measured TDOA and FDOA for the low-orbit dual-satellite passive location system is practical and imperative.

To overcome existing limitations and improve the positioning accuracy for the dual-satellite passive location system, this paper proposes a virtualization method by integrating virtual frequency conversion (VFC) and virtual reference station (VRS) [14-16] technologies to correct systematic errors in the measured FDOA and TDOA for the low-orbit dual-satellite location system. Specifically, inspired by the VRS technology in satellite navigation systems [14], the tailored VRS technology is first applied to correct the measured TDOA value by virtually establishing a reference station nearby the unknown target. Then, by considering the case where transmission frequency of reference stations may be different from that of the unknown target, the systematic error in the measured FDOA value will be corrected through integrating the proposed VRS and virtual frequency conversion technologies. Note that the proposed method can not only eliminate systematic errors in the measured TDOA and FDOA but also, to a great extent, reduce the ephemeris error. Furthermore, the proposed method needs only at least three stations to correct systematic errors. Hence, the proposed method in this paper can be regarded as a new way to correct systematic errors in the low-orbit dual-satellite passive location system. The main contributions of this paper are summarized as follows:

(1) We comprehensively analyze the coming sources of systematic errors in the low-orbit dual-satellite passive location system

(2) Inspired by the spirit of VRS, we creatively propose a new method to correct the systematic errors in the measured TDOA value

(3) The VFC method is proposed in this paper, and by combining the methods of VFC and proposed VRS, the systematic errors in the measured FDOA value is further corrected
(4) We evaluate the performance of our proposed method through extensive numerical simulations

The remainder of this paper is organized as follows. The main source of errors, which can influence the low-orbit dual-satellite passive location system, is firstly thoroughly analyzed. Then, the detailed procedures including the VRS and VFC for correcting systematic errors in the measured TDOA and FDOA are presented. After that, simulation results are illustrated to demonstrate the effectiveness of our proposed method, and finally, concluding remarks and future works are drawn. In the sequel of this paper, the superscript symbol $T$ represents transportation and $\|*\|$ represents 2-norm of vector $*$. If symbol $*$ represents a reference station or an unknown emitter, the black symbol *, without other explanations, will be its position in the Earth-Centered Earth-Fixed (ECEF) coordinated system. If $(*)$ contains systematic errors, $(*)^{0}$ is used to denote the real value of $(*)$. Since there are many symbols in this paper, the mainly used symbols are gathered in Table 1 for retrieval conveniences.

\section{Systematic Error Analysis in the Low-Orbit Dual-Satellite Location System}

In this section, we mainly study and show the error sources and types in low-orbit dual-satellite location systems. Roughly, the errors in the low-orbit dual-satellite location system could come from ephemeris errors, transponder delays, ionosphere errors, troposphere errors, multipath effects, and measured systematic errors, i.e., measured TDOA and measured FDOA. The above errors can also be classified as the spatial-correlated errors and the distanceindependent errors:

(1) Spatial-correlated errors: ephemeris errors, ionosphere errors, and troposphere errors

(2) Distance-independent errors: transponder delay, measurement systematic errors, and multipath effects

Even if there exists a number of errors affecting the positioning accuracy, we will concentrate on analyzing the errors that mainly influence the positioning accuracy in the low-orbit dual-satellite location system. Suppose that an unknown emitter on the Earth, denoted by $\mathbf{u}(X, Y, Z)$, has been measured its time of arrival (TOA), i.e., $t_{i}$, and frequency of arrival (FOA), i.e., $f_{i}$, by two satellites, whose positions are $\mathrm{S}_{i}\left(X_{i}, Y_{i}, Z_{i}\right)$ and speeds are $\dot{S}_{i}\left(\dot{X}_{i}, \dot{Y}_{i}, \dot{Z}_{i}\right)$, respectively, $i=1,2$. Then, the TOA and FOA equation of this unknown emitter $u$, measured by the master satellite, i.e., $i=1$, can be, respectively, denoted as [11] $t_{1}=1 / c \| \mathbf{u}-$ $\mathbf{S}_{1} \|$ and $f_{1}=f_{c} / c\left(\left(\mathbf{u}-\mathbf{S}_{1}\right)^{T} \dot{S}_{1} /\left\|\mathbf{u}-\mathbf{S}_{1}\right\|\right)$, where $f_{c}$ is the transmission frequency of the unknown emitter and $c$ is the signal propagation speed. Moreover, with the consideration of existing potential systematic errors, such as the clock offset on satellites, ephemeris errors, ionosphere errors, and troposphere errors, the modelled TOA and FOA measured by the master satellite could, respectively, be re-expressed as $t_{1}=1 / c\left\|\mathbf{u}-\mathbf{S}_{1}\right\|+\delta_{1}+O_{1}(\mathbf{u})+T_{1}(\mathbf{u})+I_{1}(\mathbf{u})+\varepsilon$ and $f_{1}=$ 
TABLE 1: Commonly used symbols and notations.

\begin{tabular}{|c|c|}
\hline Symbol/notation & Interpretation \\
\hline TDOA & Time difference of arrival \\
\hline FDOA & Frequency difference of arrival \\
\hline VFC & Virtual frequency conversion \\
\hline VRS & Virtual reference station \\
\hline DGPS & Differential global positioning system \\
\hline TOA & Time of arrival \\
\hline FOA & Frequency of arrival \\
\hline CVV & Combining VFC and VRS \\
\hline LEO & Low Earth orbit \\
\hline $\mathbf{u}$ & The position of the unknown emitter \\
\hline $\mathbf{S}_{i}$ & The position of the $i$ th satellite \\
\hline$\dot{\mathbf{S}}_{i}$ & The velocity of the $i$ th satellite \\
\hline$\delta_{i}$ and $\dot{\delta}_{i}$ & The total of measurement error and transponder delay of the $i$ th satellite \\
\hline$O_{i}(k)$ and $\dot{O}_{i}(\mathbf{k})$ & The ephemeris error between $i$ th satellite and unknown emitter $k$ \\
\hline$T_{i}(\mathbf{k})$ and $\dot{T}_{i}(\mathbf{k})$ & The troposphere error between $i$ th satellite and unknown emitter $k$ \\
\hline$I_{i}(\mathbf{k})$ and $\dot{I}_{i}(\mathbf{k})$ & The ionosphere error between $i$ th satellite and unknown emitter $k$ \\
\hline$\delta_{21}$ and $\dot{\delta}_{21}$ & The difference of $\delta_{i}$ or $\dot{\delta}_{i}$ on two LEO satellites \\
\hline $\mathrm{O}_{21}(\mathbf{k})$ and $\dot{O}_{21}(\mathbf{k})$ & The difference of $O_{i}(\mathbf{k})$ or $O_{i}(\mathbf{k})$ on two LEO satellites \\
\hline$T_{21}(\mathbf{k})$ and $\dot{T}_{21}(\mathbf{k})$ & The difference of $T_{i}(\mathbf{k})$ or $\dot{T}_{i}(\mathbf{k})$ on two LEO satellites \\
\hline$I_{21}(\mathbf{k})$ and $\dot{I}_{21}(\mathbf{k})$ & The difference of $I_{i}(\mathbf{k})$ or $\dot{I}_{i}(\mathbf{k})$ on two LEO satellites \\
\hline ref $_{i}$ & The position of the $i$ th reference station \\
\hline$V_{T D O A}^{i, j}$ & The comprehensive error corrections for TDOA between stations $i$ and $j$ \\
\hline$\Delta T$ & The generated error corrections of TDOA \\
\hline$\Delta f$ & The difference of transmission frequency on reference station and unknown emitter \\
\hline$F S C_{i, j}^{f_{v}}$ & The spatial-correlated error between stations $i$ and $j$ with transmission frequency $f_{v}$ \\
\hline
\end{tabular}

$\left(f_{c} / c\right)\left(\mathbf{u}-\mathbf{S}_{1}\right)^{T} \dot{S}_{1} /\left\|\mathbf{u}-\mathbf{S}_{1}\right\|+\dot{\delta}_{1}+\dot{O}_{1}(\mathbf{u})+\dot{T}_{1}(\mathbf{u})+\dot{I}_{1}(\mathbf{u})+$ $\varepsilon$, where $\delta_{1}$ and $\dot{\delta}_{1}$ represent measured systematic errors and transponder delays, respectively, $O_{1}(u)$ and $\dot{O}_{1}(u)$ are the ephemeris errors in TOA and FOA models, respectively, $I_{1}(\mathbf{u})$ and $\dot{I}_{1}(\mathbf{u})$ are ionosphere errors in TOA and FOA models, respectively, $T_{1}(\mathbf{u})$ and $\dot{T}_{1}(\mathbf{u})$ are troposphere errors in TOA and FOA models, respectively, and $\epsilon$ represents other errors in the system, such as multipath effects and some unknown errors.

Since the TOA and FOA measured by the slave satellite, i.e., $i=2$, have the same form as that of the master, the TDOA and FDOA equation of the unknown emitter $\mathbf{u}$ can be, respectively, calculated as the differences of TOA and FOA between the master and the slave satellites, which can be formulated as

$$
\begin{aligned}
& \operatorname{TDOA}(\mathbf{u})=t_{2}-t_{1}=\frac{1}{c}\left(\left\|\mathbf{u}-\mathbf{S}_{2}\right\|-\left\|\mathbf{u}-\mathbf{S}_{1}\right\|\right)+\delta_{21}+O_{21}(\mathbf{u})+T_{21}(\mathbf{u})+I_{21}(\mathbf{u})+\varepsilon, \\
& \operatorname{FDOA}(\mathbf{u})=f_{2}-f_{1}=\frac{f_{c}}{c}\left(\frac{\left(\mathbf{u}-\mathbf{S}_{2}\right)^{T} \dot{S}_{2}}{\left\|\mathbf{u}-\mathbf{S}_{2}\right\|}-\frac{\left(\mathbf{u}-\mathbf{S}_{1}\right)^{T} \dot{S}_{1}}{\left\|\mathbf{u}-\mathbf{S}_{1}\right\|}\right)+\dot{\delta}_{21}+\dot{O}_{21}(\mathbf{u})+\dot{T}_{21}(\mathbf{u})+\dot{I}_{21}(\mathbf{u})+\varepsilon,
\end{aligned}
$$

where $\delta_{21}=\delta_{2}-\delta_{1}, \dot{\delta}_{21}=\dot{\delta}_{2}-\dot{\delta}_{1}, O_{21}(\mathbf{u})$ and $\dot{O}_{21}(\mathbf{u})$ are the differences of the ephemeris error between the master and the slave satellites, $T_{21}(\mathbf{u})$ and $\dot{T}_{21}(\mathbf{u})$ are the differences of the troposphere error between the master and slave satellites, and $I_{21}(\mathbf{u})$ and $\dot{I}_{21}(\mathbf{u})$ are the differences of the ionosphere error between the master and the slave satellites.
Moreover, since the distance between the satellite and the Earth is much longer than the distance between these two low-orbit satellites, the terms $T_{21}(\mathbf{u}), \dot{T}_{21}(\mathbf{u}), I_{21}(\mathbf{u})$, and $\dot{I}_{21}(\mathbf{u})$ can all be ignored because they are much smaller [8]. Then, the TDOA and FDOA equation of the unknown emitter $u$ can be re-expressed as

$$
\begin{aligned}
& \operatorname{TDOA}(\mathbf{u})=t_{2}-t_{1}=\frac{1}{c}\left(\left\|\mathbf{u}-\mathbf{S}_{2}\right\|-\left\|\mathbf{u}-\mathbf{S}_{1}\right\|\right)+\delta_{21}+O_{21}(\mathbf{u})+\varepsilon \\
& \operatorname{FDOA}(\mathbf{u})=f_{2}-f_{1}=\frac{f_{c}}{c}\left(\frac{\left(\mathbf{S}_{2}-\mathbf{u}\right)^{T} \dot{S}_{2}}{\left\|\mathbf{u}-\mathbf{S}_{2}\right\|}-\frac{\left(\mathbf{S}_{1}-\mathbf{u}\right)^{T} \dot{S}_{1}}{\left\|\mathbf{u}-\mathbf{S}_{1}\right\|}\right)+\dot{\delta}_{21}+\dot{O}_{21}(\mathbf{u})+\varepsilon .
\end{aligned}
$$


Note that, by considering the fact that the unknown emitter is on the Earth, which can be modelled as an ellipsoid equation, the position result of the unknown emitter, i.e., $\mathbf{u}(X, Y, Z)$, can be solved by jointly considering those three equations. Furthermore, from (2) and (3), it can be seen that errors in the measured TDOA and FDOA are mainly coming from spatial-correlated errors, such as the ephemeris error, and also coming from distance-independent errors, such as the transponder delay and measured systematic errors. Note that, without considering $\delta_{21}, O_{21}(\mathbf{u})$, and $\varepsilon$ in (2) and $\dot{\delta}_{21}, \dot{O}_{21}(\mathbf{u})$, and $\epsilon$ in (3), the remaining terms in (2) and (3) are called as the theoretical TDOA and FDOA values, respectively.

\section{A Virtualization Method to Precisely Correct Errors in the Low Orbit Dual-Satellite Location System}

After completing the analyses of the coming source of errors in the low-orbit dual-satellite location system, we try to design a virtualization method to correct systematic errors in the measured TDOA and FDOA values. The proposed method to correct systematic errors consists of two folds. In Section 3.1, the tailored VRS technique is used to precisely correct the systematic error in the measured TDOA. In Section 3.2, with the consideration of the difference of transmission frequencies between reference stations and the unknown emitter, a method by combining proposed VFC and VRS, called as CVV in this paper, is proposed to correct systematic errors in the measured FDOA value.

Consider a low-orbit dual-satellite location system, as shown in Figure 1, which consists of two low-orbit satellites, six reference stations, denoted by $A, B, C, D, E, F$, whose positions are $\mathbf{r e f}_{k}, k \in(A, B, C, D, E, F)$, respectively, lying in the coverage of the low-orbit dual satellite, and an unknown emitter that locates within the area of reference stations. By measuring TDOA values of six reference stations and the unknown emitter, a VRS with its position VRS that is near the unknown emitter can be constructed. Note that, in practice, there may be more than six reference stations under the coverage of dual satellite, and the reference stations could be television towers in different provinces and also can be emitters that are set up temporarily in $a$ prior known position. In this paper, our objective is to correct systematic errors in the measured TDOA and FDOA so as to obtain a more accurate positioning result. In the following sections, for the purpose of notation conveniences, we interchangeably use the subscripts $k=1,2, \ldots$ and $k=A, B, C, D \ldots$ in this paper.

3.1. VRS Technique to Precisely Correct Errors in the Measured TDOA Value. Inspired by the VRS technique in DGPS, we are trying to apply it in the low-orbit dual-satellite location system. As shown in Figure 1, $u$ is the unknown emitter which is surrounded by several reference stations. The key problem is how to establish a virtual station nearby the unknown emitter through using these existing reference stations. Note that we observe from (3) that the spatial-

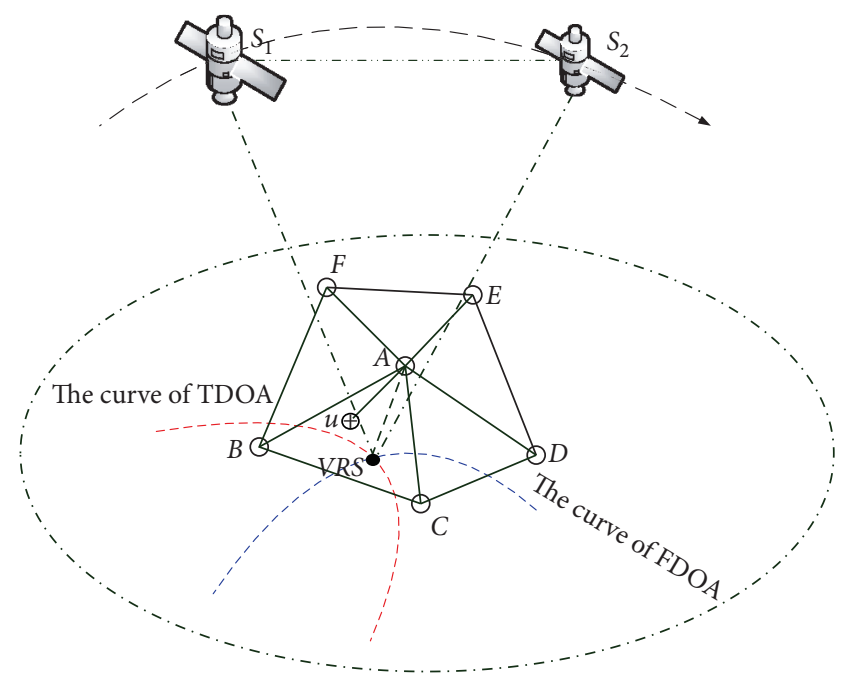

FIgURE 1: Considered system model with six reference stations in the coverage of LEO satellites.

correlated errors will remain by eliminating the distanceindependent errors through making a difference to the TDOA equations between any two reference stations. Actually, this intuition and observation provide a possibility for the realization of VRS technique in the low-orbit dualsatellite location system.

In the dual-satellite location system, VRS technique is easier to implement than in the DGPS. This is mainly because only two satellites are under consideration in the system, while in DGPS, at least four satellites are needed to solve the location, which causes more difficulty in implementing the VRS technique. Our proposed method in the first part involves the following four main steps.

3.2. The Generation of Comprehensive Error Corrections. From (2), the TDOA equation of reference station $k$ can be rewritten as

$$
\operatorname{TDOA}\left(\mathbf{r e f}_{k}\right)=\frac{1}{c}\left(r_{2}^{k}-r_{1}^{k}\right)+\delta_{21}+O_{21}\left(\mathbf{r e f}_{k}\right)+\varepsilon,
$$

where $r_{i}^{k}=\left\|\mathbf{r e f}_{k}-\mathbf{S}_{i}\right\|, i=1,2$. We further consider other two reference stations on the Earth surface, i.e., $A$ and $B$, so the difference on the TDOA equation between stations $A$ and $B$ can be expressed as

$$
\begin{aligned}
\operatorname{TDOA}^{A, B}(\mathbf{r e f}) & =\operatorname{TDOA}\left(\mathbf{r e f}_{A}\right)-\operatorname{TDOA}\left(\mathbf{r e f}_{B}\right) \\
& =\frac{1}{c}\left(r_{2}^{A}-r_{1}^{A}-r_{2}^{B}+r_{1}^{B}\right)+O_{21}^{A, B}(\mathbf{r e f})+\varepsilon,
\end{aligned}
$$

where $O_{21}^{A, B}$ (ref) is the difference of the ephemeris error between stations $A$ and $B$. Based on (5), it can be seen that the ephemeris error is the main factor to affect $\operatorname{TDOA}^{A, B}($ ref $)$, and other errors, compared to $O_{21}^{A, B}$ (ref), are much smaller, which can be almost omitted. 
Let the sum of $O_{21}^{A, B}$ (ref) and other errors be equal to $V_{\mathrm{TDOA}}^{A, B}$. Then, the comprehensive error corrections, i.e., $V_{\mathrm{TDOA}}^{A, B}$, for the TDOA equation between stations $A$ and $B$ can be

$$
V_{\mathrm{TDOA}}^{A, B}=\operatorname{TDOA}^{A, B}(\text { ref })-\frac{1}{c}\left(r_{2}^{A}-r_{1}^{A}-r_{2}^{B}+r_{1}^{B}\right) .
$$

In the low-orbit dual-satellite location system, a master reference station has to be chosen as a reference in order to implement the proposed method, and all other comprehensive error corrections should be made based on this master station. As a result, the comprehensive error corrections at the established VRS are also obtained on the basis of this chosen master station. In the following analysis, the station A, without other explanations, is chosen as the master.

3.3. The Generation of VRS Error Corrections. To make the constructed TDOA value at the VRS to be envisaged as the value that is measured by an actual physical reference station, it can be shown later that the established virtual TDOA value at the VRS can be obtained by using the comprehensive error corrections, the measured TDOA value at the master station, the geometric corrections which contain both the theoretical TDOA value at the VRS, and the theoretical TDOA value at the master station. Therefore, from (2), the TDOA equation at the master station A can be

$$
\operatorname{TDOA}\left(\operatorname{ref}_{A}\right)=\frac{1}{c}\left(r_{2}^{A}-r_{1}^{A}\right)+\delta_{21}+O_{21}\left(\operatorname{ref}_{A}\right)+\varepsilon .
$$

Likewise, the established TDOA value at the VRS has the same form as that of the master. Thus, (8) can be obtained by subtracting the TDOA equation at the VRS from that TDOA equation at station $\mathrm{A}$. Then, we have

$$
\mathrm{TDOA}^{A, \mathrm{VRS}}=\frac{1}{c}\left(r_{2}^{A}-r_{1}^{A}-r_{2}^{\mathrm{VRS}}+r_{1}^{\mathrm{VRS}}\right)+V_{\mathrm{TDOA}}^{A, \mathrm{VRS}} .
$$

After moving the term TDOA(VRS) to the left side of (8), there is

$$
\begin{aligned}
\operatorname{TDOA}(\mathbf{V R S})= & \operatorname{TDOA}\left(\mathbf{r e f}_{A}\right) \\
& -\left(\frac{1}{c}\left(r_{2}^{A}-r_{1}^{A}-r_{2}^{\mathrm{VRS}}+r_{1}^{\mathrm{VRS}}\right)+V_{\mathrm{TDOA}}^{A, \mathrm{VRS}}\right) .
\end{aligned}
$$

It is worth noting that the virtual constructed TDOA value at the VRS, i.e., TDOA(VRS), can be obtained from (9) as long as the value of $V_{\text {TDOA }}^{\text {A,VRS }}$ can be obtained. Actually, $V_{\text {TDOA }}^{\text {A,VRS }}$ can be derived from the interpolation algorithm, which will be introduced in the subsequent section. Therefore, once the comprehensive error corrections, i.e., $V_{\text {TDOA }}^{\text {A,VRS }}$, are solved, the virtual constructed TDOA value at the VRS can also be obtained.

3.4. Interpolation for Comprehensive Error Corrections. We consider a more general case by assuming that there are $N$ reference stations whose positions are ref $_{k}=\left(x_{k}, y_{k}, z_{k}\right)$, $k=1,2, \ldots, N$, separately, where the station $k=1$ is defined as the master reference station. Then, the comprehensive error corrections for TDOA between the reference $k$ and the master reference station 1 can be calculated as

$$
V_{\mathrm{TDOA}}^{1, \mathrm{VRS}}=\alpha_{1} V_{\mathrm{TDOA}}^{1,2}+\alpha_{2} V_{\mathrm{TDOA}}^{1,3}+\cdots+\alpha_{N-1} V_{\mathrm{TDOA}}^{1, N-1} .
$$

Apparently, the comprehensive error corrections between the VRS and the master station is a linear combination of others, i.e., $V_{\text {TDOA }}^{1, k}$. Moreover, the parameters $\vec{\alpha}=\left(\alpha_{1}, \alpha_{2}, \ldots, \alpha_{N}\right)^{T}$ satisfy the following conditions:

$$
\begin{aligned}
\sum_{k=1}^{N} \alpha_{i} & =1, \\
\sum_{k=1}^{N} \alpha_{i}\left(\vec{X}_{\mathrm{VRS}}-\vec{X}_{k}\right) & =1, \\
\sum_{k=1}^{N} \alpha_{i}^{2} & =\min ,
\end{aligned}
$$

where $\vec{X}_{\mathrm{VRS}}$ and $\vec{X}_{k}$ are the positions of the VRS and reference station $k$ in the Gauss plane coordinate system, respectively.

After reformulating (11) into a matrix form, the parameters $\left(\alpha_{1}, \alpha_{2}, \ldots, \alpha_{N}\right)$ can be solved by condition adjustment technology [17]. From (11), in order to get the results of those parameters, we can know that the linear combination model needs at least three reference stations, and the outcome of $\vec{\alpha}$ is related to the positions of the reference stations. Moreover, the more reference stations are added to adjust, the more accurate the $\vec{\alpha}$ in theory is. On the contrary, based on the error propagation theory [18], with the number of reference stations increasing, more errors will be introduced to $\vec{\alpha}$. Therefore, the number of reference stations to solve the parameters $\vec{\alpha}$ cannot increase all the way. There should be a trade-off between the adjustment accuracy, i.e., the result of $\vec{\alpha}$, and the number of considered reference stations. This topic is out of the scope in this paper and will be studied in our future research.

3.5. The Generation of TDOA Error Corrections for the Unknown Emitter. Since the virtual TDOA value at the VRS can be constructed by applying the previous procedures, the error corrections for the measured TDOA value for the unknown emitter can be expressed as

$$
\Delta T=\operatorname{TDOA}(\mathbf{V R S})-\left(\| \text { VRS }-\mathbf{S}_{2}\|-\| \text { VRS }-\mathbf{S}_{1} \|\right) .
$$

Note that the TDOA error corrections, i.e., $\Delta T$, contain most errors for the unknown emitter due to the fact that the position of the established VRS is very close to the position of the unknown emitter, and their errors are highly related. Therefore, $\Delta T$ can effectively correct the systematic errors of the unknown emitter. The corrected TDOA equation can be

$$
\operatorname{TDOA}(\mathbf{u})-\Delta T=\frac{1}{c}\left(\left\|\mathbf{u}-\mathbf{S}_{2}\right\|-\left\|\mathbf{u}-\mathbf{S}_{1}\right\|\right) \text {. }
$$

From the above analyses, the distance-independent error can be eliminated, and the spatial-correlated error can be 
suppressed because of the short baseline distance between the VRS and the emitter. Thus, the systematic errors in the measured TDOA value for the unknown emitter can be alleviated by applying the tailored VRS technique.

3.6. CVV Technique to Precisely Correct Errors in the Measured FDOA Value. In the previous sections, the first part of our proposed method for correcting the systematic errors in the measured TDOA value is introduced. Intuitively, is it possible to use this tailored VRS method to correct the systematic errors in the measured FDOA value. Unfortunately, the answer is no because two following troubles have to be confronted. One is that the accuracy of the constructed virtual FDOA value at the VRS is worse than that of the constructed TDOA value at the VRS. Another one is that the different transmission frequencies between reference stations and the unknown emitter will lead to a large deviation from the true FDOA value at the established VRS. Moreover, it is acknowledged that compared to the measured TDOA value, the errors in the measured FDOA value will find it more easier to degrade the positioning accuracy in the loworbit dual-satellite location system. Therefore, in order to correct the errors in the measured FDOA value, a new method, i.e., CVV, is proposed in this paper. This method consists of two steps. In the first step, VFC is used to correct the distance-independent error in the measured FDOA value, and the tailored VRS technique is employed to correct the spatial-correlated error in the second step.

3.7. VFC Method for Correcting Distance-Independent Error in the Measured FDOA Value. Without loss of generality, transmission frequencies of reference stations and the unknown emitter are given as $f_{0}$ and $f_{c}$, respectively, and they satisfy $f_{c}=f_{0}+\Delta f$, where $\Delta f$ is the difference of the transmission frequency between reference stations and the unknown emitter. Based on (3), the FDOA equation at reference station $\mathrm{A}$ with transmission frequency $f_{0}$ and the unknown emitter with transmission frequency $f_{c}$ can be, respectively, expressed as

$$
\begin{aligned}
& \operatorname{FDOA}(\mathbf{A})=\frac{f_{0}}{c}\left(\dot{r}_{2}^{A}-\dot{r}_{1}^{A}\right)+\dot{\delta}_{21}+\dot{O}_{21}(\mathbf{A}), \\
& \operatorname{FDOA}(\mathbf{u})=\frac{f_{c}}{c}\left(\dot{r}_{2}^{u}-\dot{r}_{1}^{u}\right)+\dot{\delta}_{21}+\dot{O}_{21}(\mathbf{u}) .
\end{aligned}
$$

If station $\mathrm{A}$ is chosen as the master, the method, used for correcting the measured TDOA value in the previous section, can be firstly used to eliminate the distance-independent error in (15). However, another unexpected error, caused by $\Delta f$, will be brought into the FDOA equation using the unknown emitter. In order to simultaneously reduce the distance-independent error and the ephemeris error at the unknown emitter, the method, called VFC, is proposed as follows.

Suppose that a virtual frequency $f_{v}$ is created in the loworbit dual-satellite location system. Then, by making VFC for (14) and (15), we have

$$
\begin{aligned}
& \frac{f_{v}}{f_{0}} \operatorname{TDOA}(\mathbf{A})=\frac{f_{v}}{c}\left(\dot{r}_{2}^{A}-\dot{r}_{1}^{A}\right)+\frac{f_{v}}{f_{0}} \dot{\delta}_{21}+\dot{O}_{21}^{f_{v}}(\mathbf{A}), \\
& \frac{f_{v}}{f_{c}} \operatorname{TDOA}(\mathbf{u})=\frac{f_{v}}{c}\left(\dot{r}_{2}^{u}-\dot{r}_{1}^{u}\right)+\frac{f_{v}}{f_{c}} \dot{\delta}_{21}+\dot{O}_{21}^{f_{v}}(\mathbf{u}),
\end{aligned}
$$

where $\dot{O}_{21}^{f_{v}}(\mathbf{A})$ and $\dot{O}_{21}^{f_{v}}(\mathbf{u})$, respectively, represent the ephemeris errors at reference $A$ and the unknown emitter whose transmission frequency is $f_{v}$. It is shown in Appendix that $\dot{O}_{21}$ is linear with the transmission frequency. This means that when FDOA equation is making VFC, the term $\dot{\mathrm{O}}_{21}$ only needs linear transformation.

In order to eliminate the distance-independent error, (18) can be calculated by making a difference between (16) and (17). There is

$$
\begin{aligned}
\operatorname{FDOA}^{f_{v}}(\mathbf{u})= & \operatorname{FDOA}^{f_{v}}(\mathbf{A})+\frac{f_{v}}{c}\left(\dot{r}_{2}^{u}-\dot{r}_{1}^{u}-\dot{r}_{2}^{A}+\dot{r}_{1}^{A}\right) \\
& +\left(\frac{f_{v}}{f_{c}}-\frac{f_{v}}{f_{0}}\right) \dot{\delta}_{21}+\mathrm{FSC}_{u, A}^{f_{v}} .
\end{aligned}
$$

Note that $\operatorname{FDOA}^{f_{v}}(\mathbf{u})=\left(f_{v} / f_{f}\right) \operatorname{TDOA}(\mathbf{u}), \operatorname{FDOA}^{f_{v}}$ $(\mathbf{A})=\left(f_{v} / f_{0}\right) \operatorname{FDOA}(\mathbf{A})$, and $\operatorname{FSC}_{u, A}^{f_{v}}=\dot{O}_{21}^{f_{v}}(\mathbf{u})-\dot{O}_{21}^{f_{v}}(\mathbf{A})$. From (19), the distance-independent error can be written as

$$
\left(\frac{f_{v}}{f_{c}}-\frac{f_{v}}{f_{0}}\right) \dot{\delta}_{21}=-\frac{f_{v} \Delta f_{c}}{f_{c} f_{0}} \dot{\delta}_{21} .
$$

Considering that the value of $f_{c}$ or $f_{.0}$ could be much larger than both the values of $\Delta f$ and $\delta_{21}$ in practice, a suitable virtual frequency $f_{v}$ can always be chosen to meet the inequality, i.e., $\left(f_{v} \Delta f / f_{c} f_{0}\right) \dot{\delta}_{21} \ll 1$. Therefore, (19) can be simplified to

$$
\operatorname{FDOA}^{f_{v}}(\mathbf{u})=\operatorname{FDOA}^{f_{v}}(\mathbf{A})+\frac{f_{v}}{c}\left(\dot{r}_{2}^{u}-\dot{r}_{1}^{u}-\dot{r}_{2}^{A}+\dot{r}_{1}^{A}\right)+\mathrm{FSC}_{u, A}^{f_{v}} .
$$

As shown in (20), the distance-independent error in the measured FDOA value is eliminated by the VFC, and only the spatial-correlated error is remained, i.e., $\operatorname{FSC}_{u, A}^{f_{v}}$.

3.8. VRS for Correcting Spatial-Correlated Error in the Measured FDOA Value. Note that since the comprehensive error corrections between station A and the VRS for the FDOA equation, i.e., $V_{\text {FDOA }}^{A, V R S}$, equals the sum of the spatialcorrelated error and a small systematic error, it may be possible to use $V_{\mathrm{FDOA}}^{A, \mathrm{VRS}}$ to approximate $\mathrm{FSC}_{A, \mathrm{VRS}}^{f_{v}}$. Therefore, the comprehensive error corrections can be employed to amend the spatial-correlated error in the measured FDOA value.

To successfully cancel the spatial-correlated error in the FDOA equation, another key problem is to construct the comprehensive error correction, i.e., $V_{\mathrm{FDOA}}^{\text {A,VRS }}$, whose virtual frequency is $f_{v}$ through applying the reference stations whose transmission frequency is $f_{0}$. To address this issue, the master station $\mathrm{A}$ and other adjacent stations should firstly make VFC so that all reference stations are with the 
same virtual transmission frequency of $f_{v}$. Then, by applying the abovementioned VRS technique, the comprehensive error corrections with frequency $f_{v}$ can be successfully constructed. Therefore, (21) can be amended by $V_{\mathrm{FDOA}}^{A \text {,VRS }}$, which is described as

$$
\begin{aligned}
\operatorname{FDOA}^{f_{v}}(\mathbf{u})= & \operatorname{FDOA}^{f_{v}}(\mathbf{A})+\frac{f_{v}}{c}\left(\dot{r}_{2}^{u}-\dot{r}_{1}^{u}-\dot{r}_{2}^{A}+\dot{r}_{1}^{A}\right) \\
& +\mathrm{FSC}_{u, A}^{f_{v}}-V_{\mathrm{FDOA}}^{A, \mathrm{VRS}}
\end{aligned}
$$

Since the baseline between the VRS and the unknown emitter is not far away, there will be a strong correlation between the VRS and the unknown emitter on their spatialcorrelated error. Hence, the spatial-correlated error in (21) can be corrected due to the approximate formula $\mathrm{FSC}_{u, A}^{f_{v}}-V_{\mathrm{FDOA}}^{A, \mathrm{VRS}} \approx 0$.

From the above analyses in the aforementioned sections, it turns out that the errors in the measured FDOA value can be approximately suppressed by the method of CVV. The methods for correcting the errors in the measured TDOA and FDOA values have been completely introduced; for better understanding the procedure of this method, we summarize our proposed error correction method in Figure 2.

In the following, we continue to analyze the performance of the proposed method in terms of computation complexity, which applies the computation times of the unknown variables as a metric. As for the VRS technique, it includes four main steps. In the first and second steps, they contain only $5 N$ times add operations, where $N$ is the total number of reference stations. In the third step, it needs $(N-$ 1) $N^{2}+2 N+1$ times add operations and $N^{3}+2 N+3$ times multiplication operations. In the last step, it needs 18 times add operations and 6 times multiplication operations as well as 2 times square root operations. Thus, $2 N^{3}+8 N+10$ times add operations, $N^{3}+2 N+9$ times multiplication operations, and 2 times square root operations are totally needed for the VRS technique. As for the CVV method, it includes two steps. Thus, it totally needs $(N-1) N^{2}+7 N+$ 2 times add operations and $N^{3}+4 N+3$ times multiplication operations. Therefore, the total computational complexity of our proposed method is $O\left(N^{3}\right)$.

\section{Simulation Results}

This section contains simulation results to demonstrate the theoretical development and evaluate the performance of our proposed virtualization method. In the simulation, the receivers are Low Earth Orbit (LEO) satellites with the same orbit having a distance of $800 \mathrm{~km}$ from the Earth surface, and the distance between two satellites is $50 \mathrm{~km}$, adding the ephemeris errors, respectively. Basing on the current level of the error control, the measured systematic errors for the TDOA is $80 \mathrm{~ns}$, the measured errors for the FDOA are $10 \mathrm{~Hz}$, and the transponder delay is $20 \mathrm{~ns}$. Three stations, which compose an equilateral triangle, are chosen as the reference stations in the coverage area of dual-satellite, and the baseline between separated reference stations is set to $500 \mathrm{~km}$ in our simulations. In the following analyses, as shown in Figure 1, $A$ is selected as the master and both station $B$ and $C$ are slaves. Meanwhile, in order to evaluate the performance of VRS technology, a sufficient number of selected points are regarded as the positions of the constructed VRS within this triangle.

Figure 3 is the comparison of the constructed virtual TDOA value and its corresponding true value. As shown in Figure 3, the constructed value for the TDOA at the VRS is very near to its true value, which demonstrates that the proposed method of VRS for correcting the systematic errors in the measured TDOA value is effective and can be applied in the low-orbit dual-satellite location system. Moreover, the constructed errors for TDOA value will reach the maximum of $1.8 \mathrm{~ns}$ in the area where the point numbers are located in $200 \sim 280$. This is because the selected point numbers between $200 \sim 280$ denote that the position of VRS are in the middle area of these three reference stations, which definitely degrades the performance of the VRS technique.

Since the baseline length between the constructed VRS and the unknown emitter has a remarkably influence on the correction performance for the unknown emitter, Figure 4 shows a comparison of the uncorrected and corrected errors in the measured TDOA value with different baseline lengths between the unknown emitter and the constructed VRS. As shown in Figure 4, the error after correction in the measure TDOA value is around $6.1 \mathrm{~ns}$ under the condition that the baseline is $50 \mathrm{~km}$, while the uncorrected systematic errors in the measured TDOA value at the unknown emitter is almost $100 \mathrm{~ns}$. We can see that the systematic errors in the measured TDOA can be greatly corrected at the unknown emitter, which shows the effectiveness of our tailored VRS method. Meanwhile, the correction performance is close to the ideal corrected one with the baseline increasing, and the correction performance becomes a little worse with the baseline increasing.

In order to make direct comparisons of the correction performance in the measured TDOA value by the constructed VRS, Table 2 lists the error correction efficiencies for the unknown target with different baselines. From Table 2, when the baseline is $10 \mathrm{~km}$, the TDOA correction efficiency for the unknown target is $97 \%$. Therefore, in order to decrease the baseline between the unknown emitter and the constructed VRS, the initial positioning of the unknown emitter can be determined by using the method of single station differential positioning.

Consider that since the situation where reference stations and the unknown emitter have the same transmission frequency is a special case, i.e., $\Delta f=0$, the case where reference stations and the unknown emitter have different transmission frequencies is simulated in follows, which is defined as scheme B. Besides, in order to make a comparison with the proposed method in this paper, another simulation experiment is also conducted, where the systematic error in the measured FDOA value is corrected by the VRS technique with the assumption that the reference stations and the unknown emitter always keep the same transmission frequency $f_{c}$, which is defined as scheme A. Note that scheme 
VRS for TDOA correction

The Generation of Comprehensive Error Corrections

The Generation of VRS Error Corrections

Interpolation for Comprehensive Error Corrections

The Generation of TDOA Error Corrections
CVV for FDOA correction

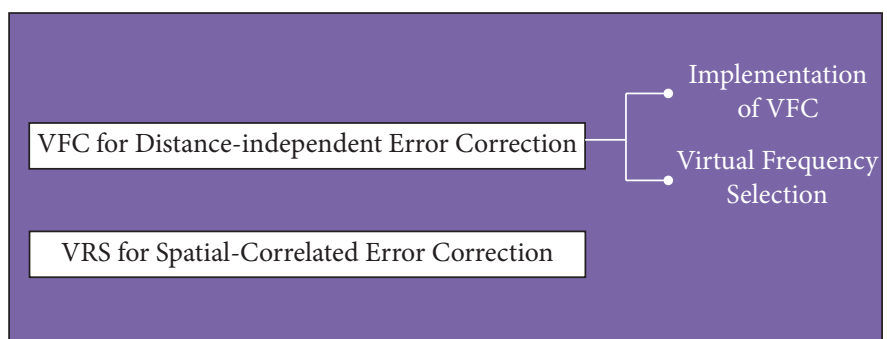

Apply the corrected TDOA and FDOA to calculate the position

FIgure 2: The flowchart of the proposed systematic error correction method.

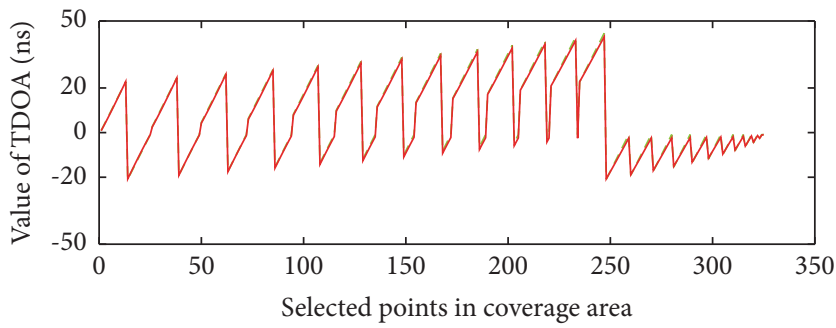

- - - The true value of TDOA at VRS

- The constructed value of TDOA at VRS

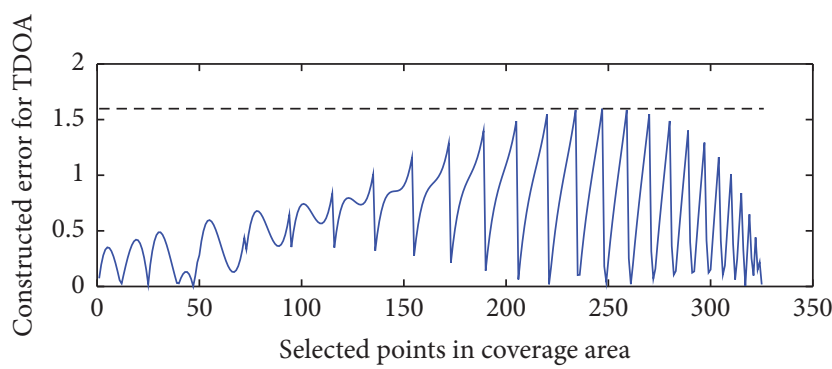

FIgure 3: Constructed accuracy for TDOA.

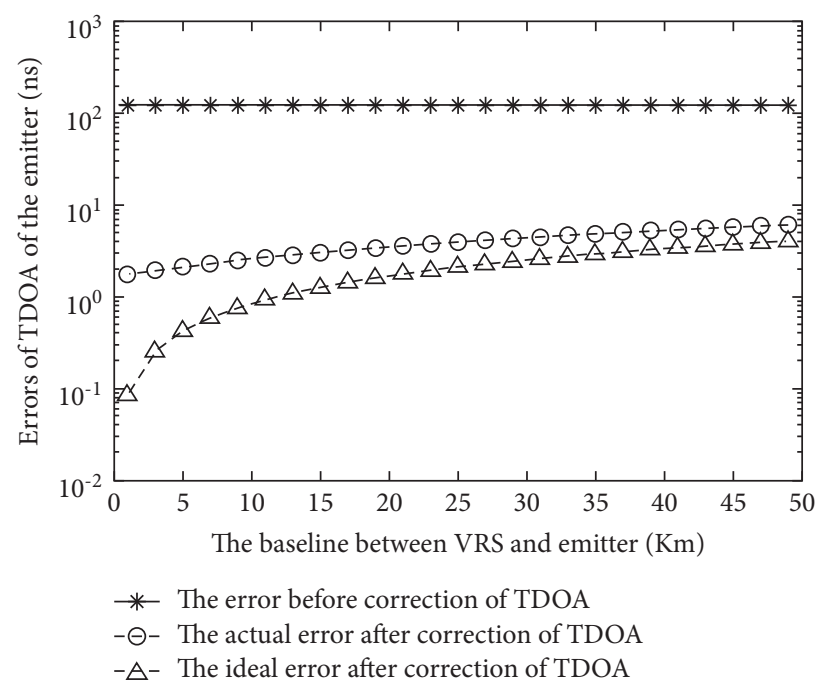

FIGURE 4: TDOA correcting accuracy of the target with the longer distance between the VRS and the unknown target. 
TABLE 2: TDOA correcting accuracy at the target with longer distance.

\begin{tabular}{lccccc}
\hline & \multirow{2}{*}{ Before correction } & $\begin{array}{c}1 \mathrm{~km} \\
\text { After correction }\end{array}$ & $\begin{array}{c}3 \mathrm{~km} \\
\text { After correction }\end{array}$ & $\begin{array}{c}10 \mathrm{~km} \\
\text { After correction }\end{array}$ & $\begin{array}{c}30 \mathrm{~km} \\
\text { After correction }\end{array}$ \\
\hline Errors of TDOA (ns) & 122.90 & 1.76 & 1.94 & 2.58 & 4.38 \\
Correction efficiency of TDOA (\%) & 0 & 98.57 & 98.42 & 97.90 & 96.44 \\
\hline
\end{tabular}
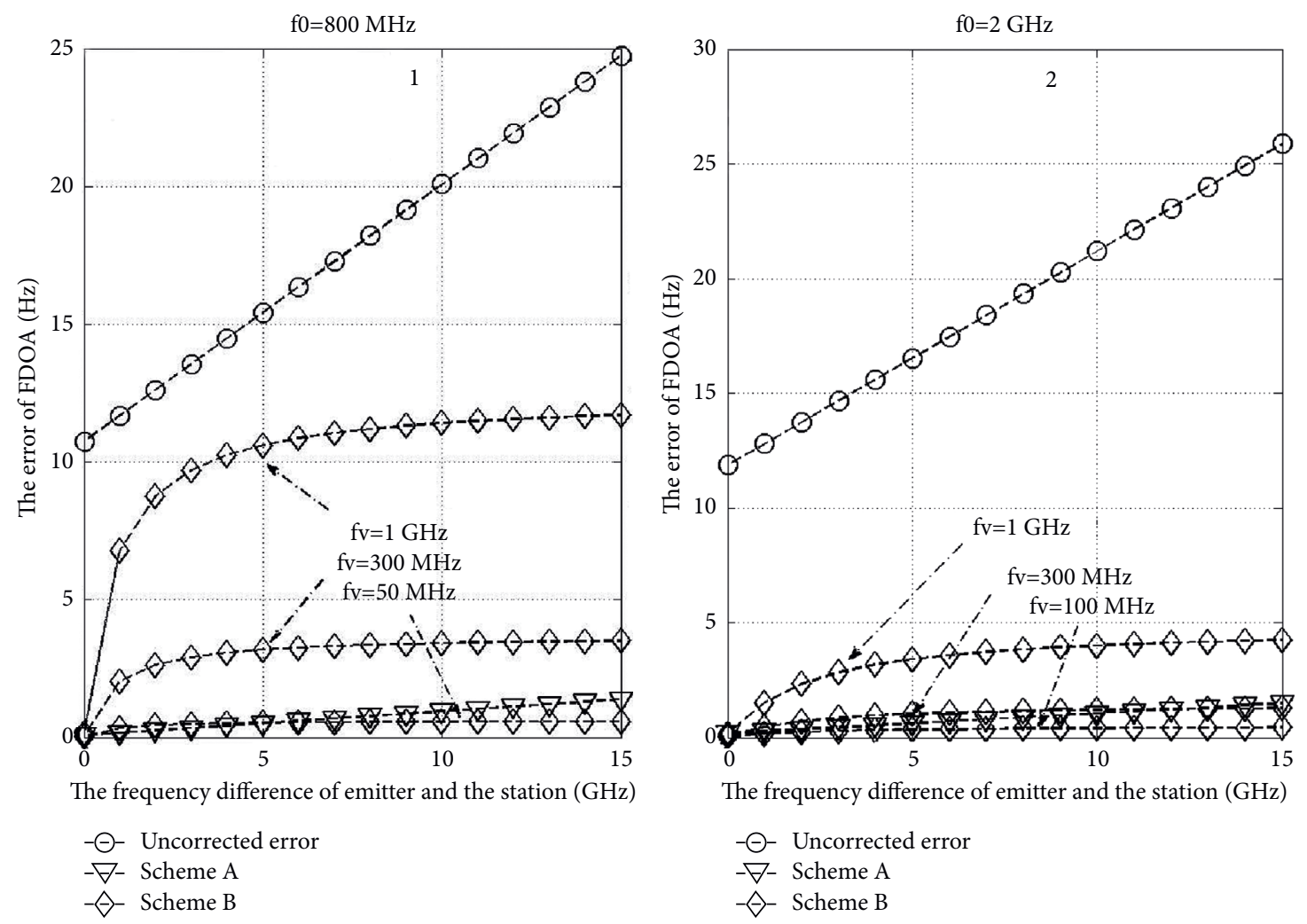

FIGURE 5: Errors in measured FDOA corrected by the proposed method.

TABle 3: Comparisons of correction efficiency in the measured FDOA value.

\begin{tabular}{|c|c|c|c|c|c|c|}
\hline \multirow[b]{2}{*}{$\Delta f(\mathrm{GHz})$} & \multicolumn{3}{|c|}{$f_{0}=800 \mathrm{MHz}$} & \multicolumn{3}{|c|}{$f_{0}=2 \mathrm{GHz}$} \\
\hline & $\begin{array}{c}\text { Before correction } \\
(\mathrm{Hz})\end{array}$ & $\begin{array}{c}\text { After correction } \\
(\mathrm{Hz})\end{array}$ & $\begin{array}{l}\text { Correction } \\
\text { efficiency }\end{array}$ & $\begin{array}{c}\text { Before correction } \\
(\mathrm{Hz})\end{array}$ & $\begin{array}{c}\text { After correction } \\
(\mathrm{Hz})\end{array}$ & $\begin{array}{l}\text { Correction } \\
\text { efficiency }\end{array}$ \\
\hline 2 & 12.61 & 2.63 & 79.15 & 13.73 & 0.70 & 94.90 \\
\hline 5 & 15.41 & 3.19 & 79.34 & 16.53 & 1.02 & 93.82 \\
\hline 8 & 19.15 & 3.36 & 82.46 & 19.33 & 1.15 & 94.05 \\
\hline 10 & 20.08 & 3.42 & 82.96 & 21.2 & 1.20 & 94.34 \\
\hline 15 & 24.75 & 3.51 & 85.82 & 24.98 & 1.27 & 94.89 \\
\hline
\end{tabular}

A is a special case, i.e., $\Delta f=0$, that hardly meets in the actual situation.

Figure 5 describes the uncorrected systematic errors in the measured FDOA value and the systematic errors after corrected by different schemes. We consider two different scenarios where the reference stations have two different transmission frequencies, i.e., $f_{0}=800 \mathrm{MHz}$ and $f_{0}$ $=2 \mathrm{GHz}$. As shown in Figure 5, the following conclusions can be drawn. (1) The uncorrected systematic error curve in the measured FDOA value is soaring with the increase of the difference of the transmission frequency between reference stations and the unknown emitter. This phenomenon can be simply explained that the transmission frequency has an influence on ephemeris error that will cause the deviation of the FDOA equation. (2) If $f_{v}$ remains unchanged, the higher the transmission frequency of reference stations, the better the correction performance for the measured FDOA value. Thus, the emitter with higher frequency should be preferred to be selected as reference stations. (3) Theoretically, no matter how large the deviation of $f_{0}$ and $f_{c}$, a suitable $f_{v}$ can 
always be found to achieve the correction performance as that in scheme A. However, a smaller chosen value of $f_{v}$ will result in smaller value of FDOA at the unknown emitter after the VFC, which may lead to worse positioning accuracy $[7,12]$. Therefore, the choice of $f_{v}$ must ensure a certain positioning accuracy.

In order to quantitatively describe the correction performance in the measured FDOA value, Table 3 lists the correction efficiency with different $\Delta f$ when the created virtual frequency is set to $300 \mathrm{MHz}$. As can be seen from Table 3, when the transmission frequency of reference stations is $800 \mathrm{MHz}$, the correction efficiency can almost reach $80 \%$. When the transmission frequency is $2 \mathrm{GHz}$, the correction efficiency can be more than $90 \%$.

\section{Conclusions}

In this paper, we first provide an analysis for the coming source of the systematic error in the low-orbit dual-satellite location system. Then, a virtualization method for precisely correcting the errors in the measured TDOA and FDOA for the low-orbit dual-satellite location system is proposed. The proposed method applies the VRS technique to correct systematic errors in the measured TDOA and leverages the proposed CVV technique to correct systematic errors in the measured FDOA. The main conclusions of this study are summarized as follows:

(1) By the leverage of the VRS technique, the constructed error for the TDOA can be the maximal value of $1.8 \mathrm{~ns}$, while the uncorrected systematic error in the TDOA is more than 100 ns. Therefore, the correction efficiency for the TDOA is $97 \%$.

(2) By applying the proposed technique of the CVV, the correction efficiency for the FDOA is related to the constructed virtual frequency and the transmission frequency of both reference stations and the unknown emitter. Moreover, when the virtual frequency is $300 \mathrm{MHz}$ and the transmission frequencies of reference stations are $800 \mathrm{MHz}$ and $2 \mathrm{GHz}$, the correction efficiencies in the measured FDOA can be about $80 \%$ and $90 \%$, respectively.

Future work can be done according to the following two aspects. (1) Although the linear combination algorithm, in this paper, can be successfully applied to generate comprehensive error corrections, the constructed accuracy for TDOA at the VRS could be further improved by exploring potential new interpolation algorithms. So, it is meaningful to figure out a more accurate interpolation algorithm. (2) The simulation results are conducted under the assumption that the layout of reference stations is equilateral triangle. However, equilateral triangle mode may not be always met in practice. It is imperative to study if other layouts of reference stations have an impact on correction performance.

\section{Appendix}

Suppose that the position and velocity of satellite $i$ are denoted by $\mathbf{S}_{\dot{0}}=\mathbf{S}_{i}^{0}+\Delta \mathbf{S}_{i}$ and $\dot{S}_{i}=\dot{S}_{i}^{0}+\Delta \dot{S}_{i}$, respectively, where $S_{i}^{0}$ and $\dot{S}_{i}^{0}$ are true position and velocity of the satellite, which are unknown to us, and $\Delta \mathbf{S}_{i}$ and $\Delta \dot{S}_{i}$ are position error and velocity error.

Let $\dot{r}_{i}^{0}$ be the true range rate between satellite $i$ and emitter $k$. It is equal to $\dot{r}_{i}^{0}=\left(\mathbf{S}_{i}^{0}-\mathbf{k}\right)^{T} \dot{S}_{i}^{0} /\left\|\mathbf{k}-\mathbf{S}_{i}^{0}\right\|$, and after expanding $\dot{r}_{i}^{0}$ around the position $\mathbf{S}_{i}$ and velocity $\dot{\mathbf{S}}_{i}$ and retaining up to the linear term of $\Delta \mathbf{S}_{i}$ and $\Delta \dot{S}_{i}$, there is $\dot{r}_{i}^{0}=\left(\mathbf{S}_{i}-\mathbf{k}\right)^{T} \dot{S}_{i} /\left\|\mathbf{k}-\mathbf{S}_{i}\right\|-D_{i}(\mathbf{k}), \quad$ where $\quad D_{i}(\mathbf{k})=1 /$ $\left\|\mathbf{S}_{i}-\mathbf{k}\right\|\left(\mathbf{S}_{i}-\left(\rho_{\mathbf{S}_{i}, \mathbf{k}}^{T} \dot{S}_{i}\right) \rho_{\mathbf{S}_{i}, \mathbf{k}}\right)^{T}+\rho_{\mathbf{S}_{i}, \mathbf{k}}^{T} \Delta \dot{S}_{i}$.

Considering a reference station $\mathrm{A}$ on the ground whose transmission frequency is $f_{0}$, the FDOA equation is $\operatorname{FDOA}(\mathbf{A})=\left(f_{0} / c\right)\left(\dot{r}_{2}^{0}-\dot{r}_{1}^{0}\right)+\delta_{21}$. After substituting $\dot{r}_{i}^{0}$ into FDOA equation, we have $\operatorname{FDOA}(\mathbf{A})=\left(f_{0} / c\right)\left(\dot{r}_{2}^{A}-\right.$ $\left.\dot{r}_{1}^{A}\right)+\dot{\delta}_{21}+\left(f_{0} / c\right)\left(D_{2}(\mathbf{A})-D_{1}(\mathbf{A})\right)$. Thus, $\dot{\mathrm{O}}_{21}^{f_{0}}$ has a linear relationship with the frequency.

\section{Data Availability}

The data used to support the findings of the study are available from the corresponding author upon request.

\section{Conflicts of Interest}

The authors declare that they have no conflicts of interest with respect to the research, authorship, and/or publication of this article.

\section{Acknowledgments}

This work was supported by the National Natural Science Foundation of China, under Grant 61671165.

\section{References}

[1] R. Bardelli, D. P. Haworth, and N. G. Smith, "Interference localization for the EUTELSAT satellite system," in Proceedings of 1995 IEEE GLOBECOM, pp. 13-17, Singapore, 1995.

[2] D. P. Haworth, N. G. Smith, R. Bardelli, and T. Clement, "Interference localization for EUTELSAT satellites-the first european transmitter location system," International Journal of Satellite Communications, vol. 15, no. 4, pp. 155-183, 1997.

[3] L. F. Duan, H. R. Lu, Y. Zhang, X. S. Guo, Y. B. Guo, and B. T. Zeng, "Accuracy analysis for passive localization from frequency measurements using single satellite," Advances in Mechanical Engineering, vol. 9, pp. 1-7, 2017.

[4] X. S. Guo, H. R. Lu, Y. Zhang, and B. T. Zeng, "Study on joint passive localization using time difference of arrival and frequency difference of arrival to improve the accuracy of foursatellite localization," Advances in Mechanical Engineering, vol. 9, no. 12, pp. 1-7, 2017.

[5] W. Geeraert, J. McMahon, and B. Jones, "Orbit determination observability of the dual-satellite geolocation system with TDOA and FDOA," in Proceedings of the AIAA/AAS Astrodynamics Specialist Conference, Long Beach, CA, USA, 2016.

[6] Z. Liu, Y. Zhao, D. Hu, and C. Liu, "A moving source localization method for distributed passive sensor using TDOA and FDOA measurements," International Journal of Antennas and Propagation, vol. 2016, Article ID 8625039, 12 pages, 2016.

[7] F. C. Guo and Y. Fan, "A method of dual-satellites geolocation using TDOA and FDOA and its precision analysis," Journal of Astronautics, vol. 29, pp. 1381-1386, 2008. 
[8] R. Xue, H. Li, X. Xu et al., "research on position differential method dual-satellites TDOA and FDOA in passive location system," in Proceedings of the Frequency Control Symposium, IEEE Computer Society, Olympic Valley, CA, USA, 2012.

[9] S. Yu, C. Cai, X. Li, S. Li, and K. Deng, "Passive location of emitter source in low orbit dual-satellites system," Lecture Notes in Electrical Engineering, vol. 20, pp. 57-70, 2013.

[10] K. C. Ho and Y. T. Chan, "Geolocation of a known altitude object from TDOA and FDOA measurements," IEEE Transactions on Aerospace and Electronic Systems, vol. 33, no. 3, pp. 770-783, 1997.

[11] J. Mason, "Algebraic two-satellite TOA/FOA position solution on an ellipsoidal earth," IEEE Transactions on Aerospace and Electronic Systems, vol. 40, no. 3, pp. 1087-1092, 2004.

[12] H. Guo, C. Guo, and Z. Li, "A method using multiple calibration for high orbit companion satellite TDOA and FDOA Geo-location system," Journal of Astronautics, vol. 33, pp. 1407-1412, 2012.

[13] Z. Qu, F. Ye, and B. Sun, "Algorithm of position calibrator for satellite interference location," Chinese Journal of Radio Science, vol. 20, pp. 342-346, 2005.

[14] C. Cai, X. Li, and H. Wu, "A virtual reference satellite differential method for relative correction of satellite ephemeris errors," Science China Physics, Mechanics \& Astronomy, vol. 53, no. 12, pp. 2300-2306, 2010.

[15] L. Herbert, V. Ulrich, and X. Chen, "Virtual reference station systems," Journal of Global Positioning Systems, vol. 1, pp. 137-143, 2002.

[16] L. Dai, W. Han, and J. Wang, "A study on GPS/GLONASS multiple reference station techniques for precise real-time carrier phase-based positioning," in Proceedings of the International Technical Meeting of the Satellite Sivision of the Instiute of Navigation, Olympic Valley, CA, USA, 2001.

[17] A. Bjerhammar, Theory of Errors and Generalized Matrix Inverses, Elservier Scientific Publishing Company Press, New York, NY, USA, 1981.

[18] M. Mikhail, Observations and Least Squares, A Dun-Donnelley Publisher Press, New York, NY, USA, 1976. 